\title{
POU1F1 is a novel fusion partner of NUP98 in acute myeloid leukemia with $\mathrm{t}(3 ; 11)(\mathrm{p} 11 ; \mathrm{p} 15)$
}

\author{
Susana Lisboa ${ }^{1 \dagger}$, Nuno Cerveira $^{1 \dagger}$, Susana Bizarro ${ }^{1}$, Cecília Correia ${ }^{1}$, Joana Vieira ${ }^{1}$, Lurdes Torres $^{1}$, José M Mariz ${ }^{2}$ \\ and Manuel R Teixeira ${ }^{1,3^{*}}$
}

\begin{abstract}
Background: NUP98 gene rearrangements have been reported in acute myeloid leukemia, giving rise to fusion proteins that seem to function as aberrant transcription factors, and are thought to be associated with poor prognosis.

Findings: A patient with treatment-related acute myeloid leukemia presented a t(3;11)(p11;p15) as the only cytogenetic abnormality. FISH and molecular genetic analyses identified a class 1 homeobox gene, POU1F1, located on chromosome 3p11, as the fusion partner of NUP98. In addition, we have found that the patient harbored an FLT3-ITD mutation, which most likely collaborated with the NUP98-POU1F1 fusion gene in malignant transformation.
\end{abstract}

Conclusions: We have identified POU1F1 as the NUP98 fusion partner in therapy-related AML with a $\mathrm{t}(3 ; 11)(\mathrm{p} 11$; p15). This is the first POU family member identified as a fusion partner in human cancer.

Keywords: NUP98 gene, NP98-POU1F1, Gene fusion, Acute leukemia

\section{Findings}

Acute myeloid leukemia (AML) is often associated with chromosomal translocations, resulting in fusion genes that have implications in disease prognosis and treatment. Chromosomal translocations involving the NUP98 gene have been reported in a wide range of hematopoietic malignancies, involving more than 20 different partner genes to generate fusion proteins with abnormal function [1]. The frequency of these rearrangements in AML is 1 to $2 \%$ and they seem to be associated with poor prognosis, thus highlighting the relevance of identifying and characterizing cases harboring such genetic alterations $[1,2]$.

The NUP98 gene codes for a protein that is a component of the nuclear pore complex (NPC) and contains multiple nontandem GLFG repeats (Gly-Leu-Phe-Gly) that are thought to function as docking sites to allow the bidirectional transport of mRNA and proteins between the nucleus and the cytoplasm [1]. The NUP98 protein is also involved in cell cycle progression, mitotic spindle

\footnotetext{
* Correspondence: manuel.teixeira@ipoporto.min-saude.pt

${ }^{\dagger}$ Equal contributors

'Department of Genetics, Portuguese Oncology Institute, Rua Dr. António Bernardino de Almeida, 4200-072 Porto, Portugal

${ }^{3}$ Institute of Biomedical Sciences (ICBAS), University of Porto, Porto, Portugal

Full list of author information is available at the end of the article
}

formation, and gene transcription [1]. All the translocations so far described involving the NUP98 gene result in the fusion of its $5^{\prime}$ region (coding for the GLFG repeats) to the $3^{\prime}$ region of the partner gene. The C-terminal partners can be divided into two general classes: homeodomain (HD) proteins and non-HD proteins [1]. HD proteins contain a DNA-binding domain (the HD domain) that is in all instances retained in the fusion protein, with its amino terminal region replaced by the GLFG repeats of NUP98. The fusion protein seems to function as an aberrant transcription factor, directly binding DNA to activate gene transcription and leading to the deregulation of HOXA cluster genes that are important for normal hematopoietic differentiation [1,3].

A 57-year-old female was diagnosed with a breast adenocarcinoma in 2001 (T1N0M0; treated with radical mastectomy, followed by four courses of chemotherapy with 5-fluorouracil, epirubicin, and cyclophosphamide, radiotherapy and hormonotherapy with tamoxifen). In 2005, the patient developed leucocytosis associated with asthenia and febrile syndrome and the diagnosis of therapy-related AML was established (AML-M4 according to the French-American-British classification). Blood count was hemoglobin $11.0 \mathrm{~g} / \mathrm{dL}$, platelets $102 \times 10^{9} / \mathrm{L}$, and leukocytes $71 \times 10^{9} / \mathrm{L}$ with $13 \%$ circulating blasts
Ciomed Central

(C) 2013 Lisboa et al.; licensee BioMed Central Ltd. This is an Open Access article distributed under the terms of the Creative Commons Attribution License (http://creativecommons.org/licenses/by/2.0), which permits unrestricted use, distribution, and reproduction in any medium, provided the original work is properly cited. 
and the bone marrow was infiltrated with $41.3 \%$ blasts. She was treated with chemotherapy (cytarabine, daunorubicin, and cyclosporin) and a complete response was attained. The patient was proposed to bone marrow transplantation, but no compatible donor was found. Ten months later the patient showed evidence of relapse with leukocytosis and thrombocytopenia and died within five months.

The bone marrow karyotype revealed a $\mathrm{t}(3 ; 11)(\mathrm{p} 11$; p15) as the sole cytogenetic abnormality in all 30 metaphases analyzed (Figure 1A), suggesting the involvement of the NUP98 gene located in 11p15. The previous knowledge that HD transcription factors are frequently involved in rearrangements with the NUP98 gene, combined with a GenBank search of putatively expressed genes on chromosomal band 3p11, prompted us to hypothesize that the NUP98 fusion partner was POU1F1. FISH analysis revealed the presence of fusion signals on the der (3) and on the der (11) chromosomes, strongly suggesting the presence of a rearrangement involving NUP98 and POU1F1 (Figure 1B).

RT-PCR with an antisense primer located on POU1F1 exon 5 and three NUP98 sense primers located on exons 9, 10, and 11 (Additional file 1) showed the presence of PCR fragments suggestive of an NUP98-POU1F1 rearrangement resulting from fusion of NUP98 exon 11 with POU1F1 exon 5 (Figure 2A). Additional RT-PCR analysis with sense primers located on NUP98 exons 9,
10, 11 and an antisense primer located on POU1F1 exon 4 and with a sense primer located on NUP98 exon 12 and antisense primers located on POU1F1 exons 4 and 5 , gave additional support to this hypothesis, since no amplification was observed (Figure 2A). Sequencing of the amplification products followed by a BLAST search confirmed that NUP98 exon 11 was fused in-frame with nucleotide 730 of the POU1F1 transcript (GenBank accession no. NM_000306) (Figure 2B). For the detection of the reciprocal POU1F1-NUP98 transcript a POU1F1 sense primer and a NUP98 antisense primer located on exons 4 and 12, respectively, were used but no fusion transcript could be detected (results not shown). This fusion is expected to give rise to a chimeric fusion protein where the N terminus of NUP98 is fused to the $\mathrm{C}$ terminus of POU1F1 (Figure 2C). This is in agreement with the results of all previously reported NUP98 fusions, in which the C-terminal DNA binding homeodomain of the homeobox protein is retained in the fusion protein and the GLFG repeats of NUP98 replace the transactivation domain [1].

For the identification of the genomic breakpoints of the NUP98-POU1F1 fusion, several primers were designed in NUP98 intron 11 and POU1F1 intron 4 (Additional file 1). When the NUP98_Fint11H sense primer was used in combination with the POU1F1_Rint4A and POU1F1_Rint4B antisense primers, amplification products of $412 \mathrm{bp}$ and 603 bp were observed, respectively (Figure 2D). Partial

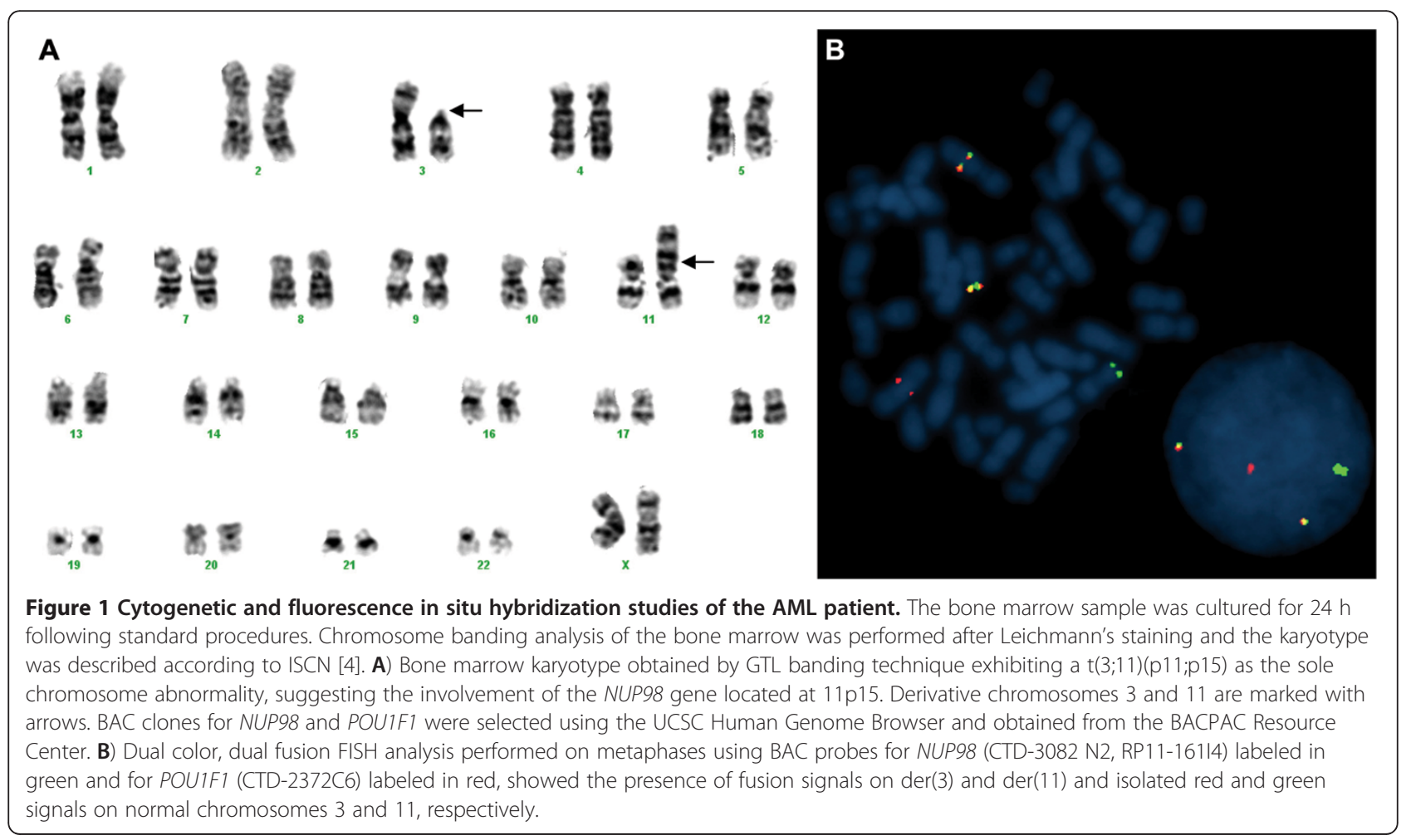




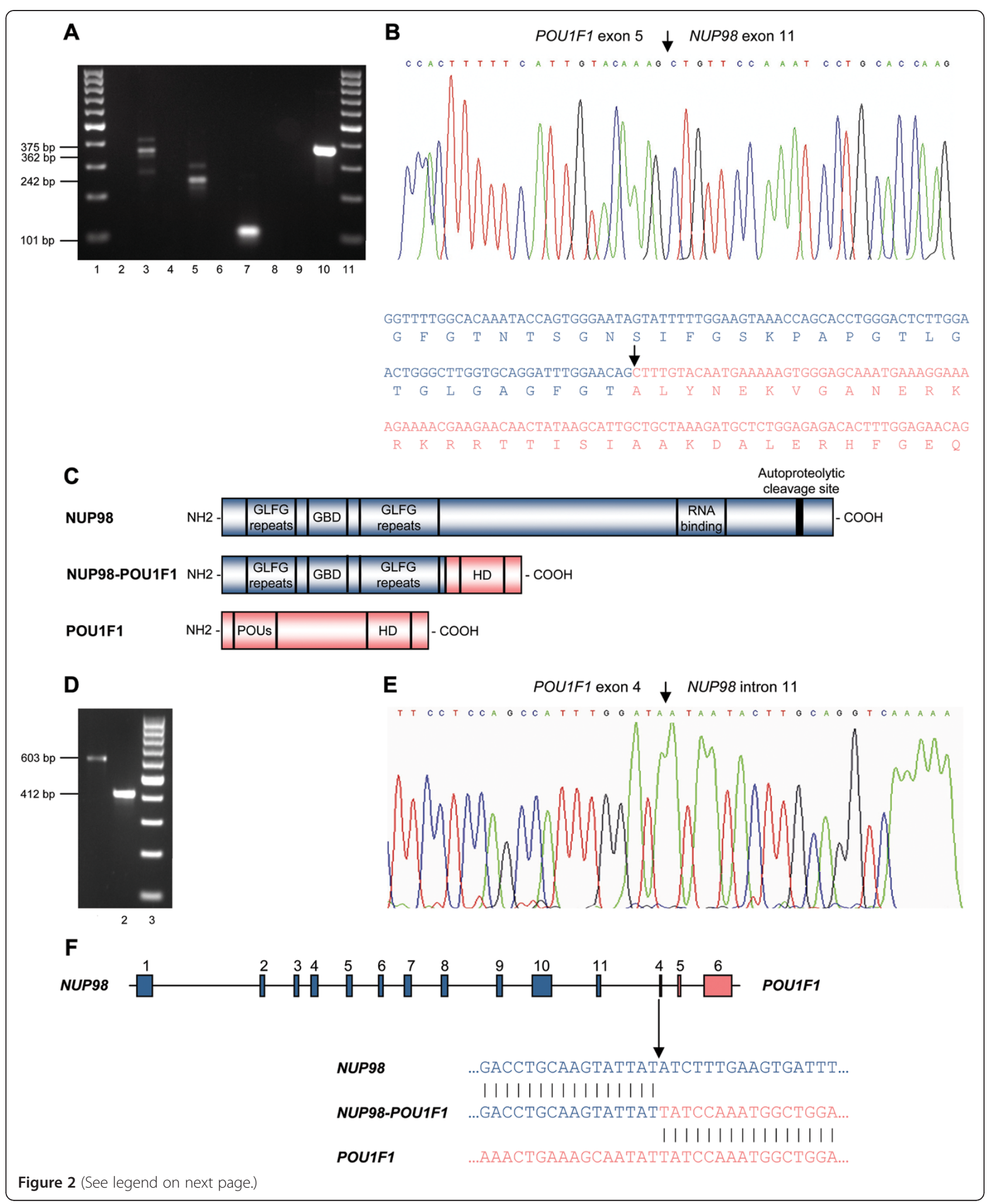


(See figure on previous page.)

Figure 2 Characterization of the NUP98-POU1F1 fusion. A) RT-PCR analysis with one antisense primer on POU1F1 exon 5 and three sense primers on NUP98 exons 9, 10, and 11 (lanes 3, 5, and 7) showed the presence of PCR fragments of 362, 242, and 101 bp, respectively, suggestive of a fusion between NUP98 exon 11 and POU1F1 exon 5. Additional RT-PCR analysis with several NUP98 and POU1F1 primer Combinations gave additional support to this hypothesis since no amplification was observed (lanes 2, 4, 6, 8, and 9). Lane 10: RNA integrity check (370 bp B2M gene fragment). Lanes 1 and 11: 100 bp molecular marker. B) Sequence analysis followed by a BLAST search confirmed that NUP98 exon 11 was fused in-frame with nucleotide 730 of the POU1F1 transcript (arrow). C) Schematic representation of the NUP98, POU1F1, and the NUP98-POU1F1 fusion proteins showing the relevant domains of the partner and chimeric proteins. D) Genomic breakpoint analysis with the NUP98_Fint11H sense primer in combination with the POU1F1_Rint4B and POU1F1_Rint4A antisense primers gave origin to amplification products of 603 and 412 bp, respectively (lanes 1 and 2). Lane 3: 100 bp molecular marker. E) Sequencing of the amplification products showed that the breakpoint was located 7490 bp downstream of NUP98 exon 11 and 129 bp downstream of the start of POU1F1 exon 4 (arrow). F) Schematic representation of the genomic DNA breakpoint (arrow) and nucleotide sequence of the genomic breakpoint of the translocation $\mathrm{t}(3 ; 11)$ and corresponding normal chromosomes 3 and 11 .

sequencing of the amplification products showed that the breakpoint was located 7490 bp downstream of NUP98 exon 11 and, interestingly, within POU1F1 exon 4, 129 bp downstream of the start of POU1F1 exon 4, and no evidence of mutation or deletion was detected in the breakpoint region (Figure $2 \mathrm{~A}$ and $\mathrm{F}$ ). This leads to retention in the genomic sequence of 36 nucleotides from POU1F1 exon 4 that are not included in the mature NUP98POU1F1 messenger RNA, probably as a result of the removal of the splice acceptor site of POU1F1 intron 3.

Since FLT3-ITD mutations have been reported in more than $50 \%$ of the patients with NUP98-HOX fusions [5], we have searched for this abnormality in our patient using the FLT3 Mutation Assay for Gel Detection (InVivoScribe Technologies, San Diego, USA) according to the manufacturer's instructions. We found an internal tandem mutation of the FLT3 gene, as shown by the presence of an amplification product of approximately $350 \mathrm{bp}$ (Additional file 2: Figure S1).

To our knowledge, this is the first time that POU1F1, a POU class 1 homeobox gene, is reported as being involved in a fusion gene in human cancer. POU1F1 belongs to the POU family of transcription factors that plays a fundamental role in inhibition and promotion of cell differentiation, as well as in the determination of cell lineage and regulation of cell migration, survival and terminal differentiation [6]. In adults, POU1F1 is expressed in cells of the anterior pituitary gland, where it plays a role in cellular commitment, differentiation and proliferation, driving the expression of growth hormone, prolactin, and thyroid-stimulating hormone $\beta$ chain genes [6]. POU1F1 expression has also been reported in hematopoietic and lymphoid tissues [7], and its expression was correlated with increased cellular proliferation in breast cancer and human myeloid leukemic cells, leading to the suggestion that POUF1 may be involved in the regulation of cellular proliferation [8-10]. Since the expression of NUP98-HOX chimeric genes seems to be under the control of the NUP98 promoter [1], leading to overexpression of the $\mathrm{HD}$ transcription factor, it is expected that the same occurs in the rearrangement we here describe, and that POU1F1 overexpression might result in increased proliferation of leukemic cells.

Several lines of evidence have suggested that many of the NUP98 fusion proteins can act as aberrant transcription factors. It seems that a synergistic action between NUP98 and the HD partner gene leads to the creation of a unique protein with unique DNA targeting properties and function, which can lead to leukemogenic transformation [1]. Indeed, the NUP98 chimeric proteins not only retain the $\mathrm{N}$-terminal sequences that are responsible for both DNA binding and transcription activation through a "cryptic" transactivation domain [11-13], but also the C-terminal region of the HD partner can directly bind DNA and activate gene transcription [1]. Furthermore, mouse models of NUP98-HOX fusions were shown to induce leukemia with variable latency [1], which was associated with deregulation of HOXA cluster genes that are thought to play a key role in normal hematopoietic differentiation [14]. Impaired terminal differentiation of hematopoietic cells is a hallmark of leukemia and, according to the two-hit model of leukemogenesis, is classified as a type II mutation [15]. However, this model of leukemic transformation, although overly simplified, requires the presence of a concomitant mutation leading to increased proliferation, survival, or both (type I mutation) [15]. It seems that NUP98-HOX fusions have the ability to initiate and maintain a state of self-renewal necessary, but not sufficient, for the development of leukemia [1]. Indeed, type I mutations are common in NUP98 rearranged leukemia, including mutations in the NRAS, KRAS, KIT, WT1 and FLT3 genes [5,16]. The type I mutation in the case we here present was the FLT3-ITD mutation, which we hypothesize collaborated with the NUP98POU1F1 fusion in malignant transformation.

In summary, we have identified POU1F1 as the NUP98 fusion partner in therapy-related AML with a $\mathrm{t}(3 ; 11)(\mathrm{p} 11 ; \mathrm{p} 15)$. This is the first POU family member identified as a fusion partner in leukemia, and further studies are necessary to uncover the precise role played by this family of genes in this disease. 


\section{Additional files}

Additional file 1: Oligonucleotide primers used in this study.

Additional file 2: Figure S1. Detection and analysis of the FLT3-ITD mutation. Lane 1 and 8: 100 bp molecular marker. Lane 2: specimen control size ladder with amplification products of approximately 100, 200, 300,400 and 600 bp confirming the patient DNA sample integrity. Lanes 3 and 7: no template controls. Lane 4: presence of amplification products of approximately $330 \mathrm{bp}$ (the wild type allele) and a larger amplification product corresponding to the detection of internal tandem duplication (ITD) of the FLT3 gene. Lane 5: negative control (amplification of polyclonal control DNA of approximately $330 \mathrm{bp}$ ). Lane 6: positive control (amplification of clonal control DNA of approximately $360 \mathrm{bp}$ ).

\section{Competing interests}

The authors declare that they have no competing interests.

\section{Authors' contributions}

$\mathrm{SL}$ and NC designed and performed the research, analyzed the data and drafted the manuscript. SB performed the sequencing and mutation analyses. CC, JV, and LT performed the chromosome banding and molecular cytogenetic studies. JMM clinically assessed the patient. MRT coordinated the study and participated in manuscript writing. All authors read and approved the final manuscript.

\section{Acknowledgements}

This work was supported by a grant from the "Associação Portuguesa Contra a Leucemia" (2006-30.2.AP/MJ) and "Liga Portuguesa Contra o Cancro".

\section{Author details}

${ }^{1}$ Department of Genetics, Portuguese Oncology Institute, Rua Dr. António Bernardino de Almeida, 4200-072 Porto, Portugal. Department of Onco-Hematology, Portuguese Oncology Institute, Porto, Portugal. ${ }^{3}$ Institute of Biomedical Sciences (ICBAS), University of Porto, Porto, Portugal.

Received: 12 September 2012 Accepted: 3 January 2013

Published: 18 January 2013

\section{References}

1. Gough SM, Slape Cl, Aplan PD: NUP98 gene fusions and hematopoietic malignancies: common themes and new biologic insights. Blood 2011, 118:6247-6257.

2. Nebral K, König M, Schmidt HH, Lutz D, Sperr WR, Kalwak K, Brugger S, Dworzak MN, Haas OA, Strehl S: Screening for NUP98 rearrangements in hematopoietic malignancies by fluorescence in situ hybridization. Haematologica 2005, 90:746-752.

3. Eklund $E$ : The role of Hox proteins in leukemogenesis: insights into key regulatory events in hematopoiesis. Crit Rev Oncog 2011, 16:65-76.

4. Shaffer LG, Slovak ML, Campbell LJ: An International System for Human Cytogenetic Nomenclature. Basel: Karger Publishers; 2009.

5. Chou WC, Chen CY, Hou HA, Lin LI, Tang JL, Yao M, Tsay W, Ko BS, Wu SJ, Huang SY, et al: Acute myeloid leukemia bearing $t(7 ; 11)(p 15 ; p 15)$ is a distinct cytogenetic entity with poor outcome and a distinct mutation profile: comparative analysis of 493 adult patients. Leukemia 2009, 23:1303-1310.

6. Andersen B, Rosenfeld MG: POU domain factors in the neuroendocrine system: lessons from developmental biology provide insights into human disease. Endocr Rev 2011, 22:2-35.

7. Delhase M, Vergani P, Malur A, Hooghe-Peters EL, Hooghe RJ: The transcription factor Pit-1/GHF-1 is expressed in hematopoietic and lymphoid tissues. Eur J Immunol 1993, 23:951-955.

8. Ben-Batalla I, Seoane S, Garcia-Caballero T, Gallego R, Macia M, Gonzalez LO, Vizoso F, Perez-Fernandez R: Deregulation of the Pit-1 transcription factor in human breast cancer cells promotes tumor growth and metastasis. J Clin Invest 2010, 120:4289-4302.

9. Ben-Batalla I, Seoane S, Macia M, Garcia-Caballero T, Gonzalez LO, Vizoso F, Perez-Fernandez R: The Pit-1/Pou1f1 transcription factor regulates and correlates with prolactin expression in human breast cell lines and tumors. Endocr Relat Cancer 2010, 17:73-85.
10. Costoya JA, García-Barros M, Gallego R, Señarís R, Arce VM, Devesa J: Correlation of Pit-1 gene expression and Pit-1 content with proliferation and differentiation in human myeloid leukemic cells. Exp Cell Res 1998, 245:132-136.

11. Capelson M, Liang Y, Schulte R, Mair W, Wagner U, Hetzer MW: Chromatinbound nuclear pore components regulate gene expression in higher eukaryotes. Cell 2010, 140:372-383.

12. Kalverda B, Pickersgill H, Shloma W, Fornerod M: Nucleoporins directly stimulate expression of developmental and cell-cycle genes inside the nucleoplasm. Cell 2010, 140:360-371.

13. Xu S, Powers MA: Nup98-homeodomain fusions interact with endogenous Nup98 during interphase and localize to kinetochores and chromosome arms during mitosis. Mol Biol Cell 2010, 21:1585-1596.

14. Argiropoulos B, Humphries RK: Hox genes in hematopoiesis and leukemogenesis. Oncogene 2007, 26:6766-6776.

15. Kosmider O, Moreau-Gachelin F: From mice to human: the "two-hit model" of leukemogenesis. Cell Cycle 2006, 5:569-570.

16. Taketani T, Taki T, Nakamura T, Kobayashi Y, Ito E, Fukuda S, Yamaguchi S, Hayashi Y: High frequencies of simultaneous FLT3-ITD, WT1 and KIT mutations in hematological malignancies with NUP98-fusion genes. Leukemia 2010, 24:1975-1977.

\section{doi:10.1186/1476-4598-12-5}

Cite this article as: Lisboa et al:: POU1F1 is a novel fusion partner of NUP98 in acute myeloid leukemia with $\mathrm{t}(3 ; 11)(\mathrm{p} 11 ; \mathrm{p} 15)$. Molecular Cancer 2013 12:5.

\section{Submit your next manuscript to BioMed Central and take full advantage of:}

- Convenient online submission

- Thorough peer review

- No space constraints or color figure charges

- Immediate publication on acceptance

- Inclusion in PubMed, CAS, Scopus and Google Scholar

- Research which is freely available for redistribution 